\title{
Antibacterial Porous Polymeric Monolith Columns with Amphiphilic and Polycationic Character on Cross-linked PMMA Substrates for Cell Lysis Applications
}

\author{
Mohamed Aly Saad Aly, Olivier Nguon, Mario Gauthier and John T. W. Yeow*
}

\author{
Received Xth XXXXXXXXXX 20XX, Accepted Xth XXXXXXXXX 20XX \\ First published on the web Xth $X X X X X X X X X X 200 X$ \\ DOI: $10.1039 / b 000000 x$
}

The application of porous polymeric monolith (PPM) columns as an effective tool for bacterial cell lysis within microfluidic chips is demonstrated. By taking advantage of the large surface area and controllable pore size inherent to PPMs, we developed a double mechanism cell lysis technique. The bacterial cell wall is mechanically sheared by flowing through the porous medium of the PPM column, but it is also damaged and disintegrated by physical contact with the antibacterial polymeric biocide covering the porous surface. This leads to leakage of the intracellular contents. The stable and non-leaching antibacterial column introduced in this work alleviates the need for chemical or enzymatic lysins and their potential release of polymerase chain reaction (PCR) inhibitors. The PPM columns were obtained by the photoinitiated free radical copolymerization of n-butyl methacrylate (BuMA) and N-(tert-butyloxycarbonyl)aminoethyl methacrylate (Boc-AEMA) in the presence of a cross-linker and porogenic solvents. The porous network was synthesized directly inside a microfluidic channel fabricated in a cross-linked poly(methyl methacrylate) (X-PMMA) substrate by laser micromachining. After removing the Boc protecting group with phosphoric acid, an amphiphilic and cationic network structure reminiscent of synthetic mimics of antimicrobial peptides (SMAMPs) was obtained. The antibacterial activity of the PPM columns was tested against Bacillus subtilis (B. subtilis) and Escherichia coli (E. coli) cells. Cell lysis was evidenced by DNA release, which was then amplified by PCR and confirmed by gel electrophoresis, to verify that the antibacterial monolithic columns did not strongly interfere with the PCR process.

\section{Introduction}

Porous polymeric monoliths (PPMs) were introduced over the last decade, and are mainly used in liquid chromatography and biological substances extraction. PPMs are formed within the boundaries of a closed volume such as capillaries and microfluidic channels, by free radical polymerization of a mixture that includes functional monomers, a cross-linking monomer, a free-radical initiator, and porogenic solvents. Recently PPMs were utilized for cell lysis within microchips, as demonstrated by Mahalanabis et al., ${ }^{1}$ in which the bacterial cells were lysed by mechanical shearing of the cell walls through flowing in a PPM column within a microfluidic channel assisted with detergent lytic conditions. The same group impregnated multi-walled carbon nanotubes (MWCNT) into the PPMs and claimed that this approach improved the lysis efficiency, albeit with chemical and enzymatic pretreatment of the bacterial cells. ${ }^{2}$

Antimicrobial polymers, often called polymeric biocides, are polymers possessing antimicrobial properties, i.e. the

University of Waterloo, 200 University Ave West, Waterloo, Ontario, Canada N2L 3G1. E-mail: jyeow@uwaterloo.ca; Fax: +1 519 746-4791; Tel: +1 519 888-4567 Ext. 32152 ability to inhibit the growth and eventually kill microorganisms such as bacteria and fungi. Research is ongoing to engineer these polymers so as to imitate natural host defense peptides (HDPs) used by the immune systems in living organisms to kill bacteria. That emerging class of antimicrobial polymers, termed 'synthetic mimics of antimicrobial peptides' (SMAMPs), ${ }^{3}$ SMAMPs is synthesized to mimic the main features of HDPs: cationic charge and amphiphilic character, which lead to the permeation and then the disintegration of the bacterial membrane. An antimicrobial surface is a form of antimicrobial polymers killing cells by contact. Tiller et $a l .{ }^{4}$ introduced in 2001 surfaces that killed bacteria upon contact, and termed it 'contact killing'. Antibacterial polymers have been attached to different surfaces by numerous techniques including chemical grafting, ${ }^{5},{ }^{6}$ layer-by-layer deposition, ${ }^{7},{ }^{8}$ and grafting from. ${ }^{9}$ Madkour et al. ${ }^{9}$ thus used surface-initiated atom transfer radical polymerization (ATRP) to grow an antibacterial copolymer, poly(butyl methacrylateco-aminoethyl methacrylate), from surfaces. These displayed a high antimicrobial activity that killed $100 \%$ of S. aureus and E. coli in less than 5 minutes.

In this paper we report for the first time on the preparation of porous polymeric monoliths with antibacterial surfaces 
within a microfluidic chip, to form an antibacterial porous structure belonging to the SMAMPs family. We fabricated antimicrobial PPM columns by in situ photoinitiated free radical copolymerization of n-butyl methacrylate (BuMA) and $\mathrm{N}$-(tert-butyloxycarbonyl)aminoethyl methacrylate (BocAEMA), cross-linked with ethylene glycol dimethacrylate (EGDMA) in the presence of porogenic solvents. In the PPM developed, bacterial cells undergo lysis through a dual mechanism: mechanical lysis, by forcing bacterial cells to flow into the porous medium of the PPM column, and contact killing, when the cells come in contact with the antibacterial surface of the column. The non-leaching nature of the antibacterial structure yields a lysate ready to use for PCR. We also demonstrate the use of X-PMMA as a substrate for the microfluidic channel that covalently bonds with the monolithic column through the unreacted $\mathrm{C}=\mathrm{C}$ double bonds on the surface of X-PMMA. This approach eliminates the need of surface functionalization to create a bonding intermediate layer between the substrate and the PPM column.

\section{Experimental}

\subsection{Materials}

Methyl methacrylate (MMA, 99\%), butyl methacrylate (BuMA, $99 \%$ ), and ethylene glycol dimethacrylate (EGDMA, 98\%) were purchased from Sigma-Aldrich (Oakville, ON Canada), and filtered over alumina prior to use to remove inhibitors. Dichloromethane (DCM, 99.9\%, Chromasolv Plus), tetrahydrofuran (THF, 99.9\%, Chromasolv Plus) and triethylamine (TEA, 99.5\%) were also purchased from SigmaAldrich and were distilled prior to use. Benzoyl peroxide (BPO, 97\%), N,N-diisopropylethylamine (DIPEA, 99\% Reagent Plus), methacryloyl chloride (MACl, 97\% purum), 1dodecanol (98\%, reagent grade), cyclohexanol (99\% Reagent Plus), 2,2-dimethoxy-2-phenylacetophenone (DMPAP, 99\%) and methanol ( $99.9 \%$ Chromasolv) were purchased from Sigma-Aldrich and used without further purification. 2Aminoethyl methacrylate hydrochloride (AEMA-HCl, 95\%) was purchased from Polysciences Inc. (Warrington, PA USA) and was used without further purification. Fumed silica (powder, 0.2-0.3 $\mu \mathrm{m}$ avg. part size), phosphoric acid (85\%), 1,4-dioxane (anhydrous, 99.8\%), trifluoroacetic acid (TFA, 99\%) and potassium bromide (ACS reagent, 99.0\%, $\mathrm{KBr}$ ) were purchased from Sigma-Aldrich and used as received. iTaq polymerase, 10X PCR buffer, and magnesium chloride were obtained from Biorad. Primers, and dNTP mix (dATP, dCTP, dGTP and dTTP) were obtained from SigmaAldrich.Ethidium bromide (UltraPure $10 \mathrm{mg} / \mathrm{ml}, \mathrm{EtBr}$ ) was purchased from Life Technologies Inc. (Burlington, ON Canada). 100 bp DNA ladder was purchased from BioLabs.

\subsection{Synthesis of cross-linked PMMA}

MMA and EGDMA were filtered over a thin layer of basic aluminum oxide (alumina) and added to a dry Erlenmeyer flask. The total reaction volume was set at $150 \mathrm{~mL}$, and the amount of EGDMA was adjusted to either 3, 5, 10, 15, or 20 mole \% as shown in Table 1. After the addition of BPO (2.30 g, 9.5 $\mathrm{mmol}, 0.75 \mathrm{~mol} \%$ ) the mixture was degassed for $1 \mathrm{~h}$ under $\mathrm{N}_{2}$ flow. The solution was transferred with a canula to a dry soap-washed crystallizing dish $\left(190 \times 100 \mathrm{~cm}^{2}\right)$ covered with aluminum foil and kept under nitrogen atmosphere. The dish was immersed in a water bath at $35^{\circ} \mathrm{C}$ and leveled to insure a uniform substrate thickness. The solution was kept for 1.5 $\mathrm{h}$ under nitrogen before turning off the water bath heater. As the polymerization reaction was exothermic, cold water was added to the water bath to avoid overheating, and then the dish was kept under nitrogen at room temperature for $11 \mathrm{~h}$. The transparent cross-linked PMMA substrate was washed by soaking in methanol for $30 \mathrm{~min}$, and dried under air flow. The polymer was easily removed from the dish with a spatula. The X-PMMA substrate was then cut into $40 \times 20 \mathrm{~mm}^{2}$ slices with $1 \mathrm{~mm}$ thickness using a laser ablation machine. With a spectrophotometer, the absorbance of the X-PMMA samples at $365 \mathrm{~nm}$ wavelength was determined to be 0.238 .

Table 1 MMA and EGDMA volumes used in the preparation of the different cross-linked PMMA (X-PMMA) substrates.

\begin{tabular}{lll}
\hline Cross-linker (mole \%) & MMA $(\mathrm{mL})$ & EGDMA $(\mathrm{mL})$ \\
\hline 3 & 145.6 & 4.4 \\
5 & 142.9 & 7.1 \\
10 & 136.4 & 13.6 \\
15 & 130.4 & 19.6 \\
20 & 125.0 & 25.0 \\
\hline
\end{tabular}

\subsection{Microchip fabrication}

Microfluidic channels were micromachined on the X-PMMA substrates with a $10.6 \mu \mathrm{m} \mathrm{CO}_{2}$ laser engraving system (Universal Laser Systems, VLS2.30). In order to obtain an enclosed channel, another piece of the X-PMMA substrate in which two holes were drilled for the inlet and outlet was chemically bonded with the substrate hosting the microchannel. A thin layer of BuMA was applied between the two X-PMMA layers to enhance bonding between the two networks. Thermal bonding was achieved by placing the top and bottom substrates under pressure in a vise press and in a pre-heated oven at $130{ }^{\circ} \mathrm{C}$ for $30 \mathrm{~min}$. Two $30 \mathrm{G}$ syringe needles were trimmed and placed over the inlet and outlet holes. They were set with epoxy glue mixed with fine fumed silica powder, to achieve a hard and stable adhesive layer. 


\subsection{Antibacterial polymer synthesis and characterization}

2.4.1 Boc-EA synthesis. The synthetic procedure shown in Scheme 1 was adapted from Fedotenko et al. ${ }^{10}$ Briefly, distilled triethylamine (TEA, $19.2 \mathrm{~mL}, 138 \mathrm{mmol}$ ) was added to a solution of 2-aminoethanol $(5.42 \mathrm{~mL}, 89.7 \mathrm{mmol})$ in 200 $\mathrm{mL}$ of dry dichloromethane (DCM) and stirred at room temperature for $30 \mathrm{~min}$ before cooling to $0^{\circ} \mathrm{C}$. A solution of ditert-butyl dicarbonate $\left(\mathrm{BOC}_{2} \mathrm{O}, 20 \mathrm{~g}, 89.7 \mathrm{mmol}\right)$ in $50 \mathrm{~mL}$ of dry DCM was then added drop-wise. The reaction mixture was stirred at room temperature for $10 \mathrm{~h}$, and quenched with a saturated aqueous solution of ammonium chloride $\left(\mathrm{NH}_{4} \mathrm{Cl}\right)$. Liquid extraction with $100 \mathrm{~mL}$ of DCM was repeated 3 times, and the combined organic fractions were washed with brine. After drying over $\mathrm{Na}_{2} \mathrm{SO}_{4}$, the solvent was evaporated under reduced pressure. The crude product was purified on a silica gel column ( $2 \%$ methanol/DCM) and yielded a clear liquid (9 g, $55.8 \mathrm{~mol}, 62 \%)$. Rf: $0.29 .{ }^{1} \mathrm{H}$ NMR (300 $\left.\mathrm{MHz} \mathrm{CDCl}_{3}\right): \delta$ 5.268 (br s, 1H), 3.647 (s, 2H), 3.238 (s, 2H), 3.007 (br s, 1H), $1.409(\mathrm{~s}, 9 \mathrm{H}),{ }^{13} \mathrm{C} \mathrm{NMR}\left(300 \mathrm{MHz}, \mathrm{CDCl}_{3}\right): \delta 167.166, \delta$ $155.629, \delta 135.920, \delta 126.776, \delta 79.447, \delta 63.823, \delta 39.573$, $\delta 28.230, \delta 18.160$.

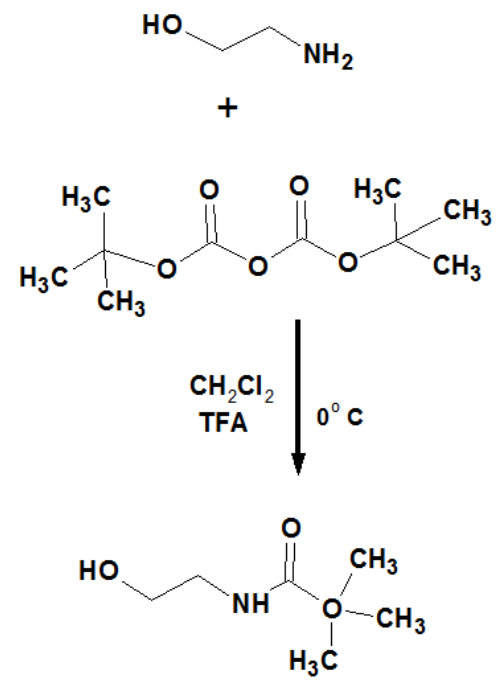

Scheme 1. Synthesis of Boc-EA.

2.4.2 Boc-EAMA synthesis. The synthetic procedure shown in Scheme 2 was adapted from Kuroda et al. ${ }^{11}$ BocEA, DCM and DIPEA were added to an oven-dried roundbottom flask, and the solution was degassed with nitrogen flow for $20 \mathrm{~min}$. The flask was then cooled in a dry ice/acetone bath and $\mathrm{MACl}$ was added drop-wise with vigorous stirring under nitrogen atmosphere. After $15 \mathrm{~min}$, the solution was brought to room temperature and allowed to react overnight. The light purple solution was washed successively with water,
$10 \%\left(\mathrm{w} / \mathrm{v}\right.$ ) citric acid, $10 \%(\mathrm{w} / \mathrm{v}) \mathrm{K}_{2} \mathrm{CO}_{3}, 9 \%(\mathrm{w} / \mathrm{v}) \mathrm{NaHCO}_{3}$, and saturated $\mathrm{NaCl}$ aqueous solutions. The bottom organic layer was dried over $\mathrm{Na}_{2} \mathrm{SO}_{4}$, and the solvent was evaporated under reduced pressure. Recrystallization from DCM/hexanes yielded clear crystals $\left(22.4 \mathrm{~g}, 61 \%\right.$ yield). ${ }^{1} \mathrm{H}$ NMR (300 $\mathrm{MHz}, \mathrm{CDCl} 3$ ): $\delta 6.090(\mathrm{~s}, 1 \mathrm{H}), 5.552$ (s, 1H), 4.768 (br s, $1 \mathrm{H}), 4.170(\mathrm{t}, 2 \mathrm{H}), 3.405$ (br, 2H), $1.915(\mathrm{~s}, 3 \mathrm{H}), 1.410(\mathrm{~s}$, 9H). 13C NMR (300 MHz, CDCl3): $\delta$ 167.166, $\delta$ 155.629, $\delta$ $135.920, \delta 126.776, \delta 79.447, \delta 63.823, \delta 39.573, \delta 28.230$, $\delta 18.160$.<smiles>C=C(C)C(=O)C(=O)OCCNC(=O)OC(C)(C)OC(=O)NCCO</smiles>

Scheme 2. Synthesis of Boc-EAMA.

2.4.3 Network fabrication and deprotection. The composition used to prepare the antibacterial network was first investigated off-chip, by mixing $0.3 \mathrm{~g}$ of Boc-AEMA (30 wt \%), $0.25 \mathrm{~g}$ of BuMA (2.5 wt \%), $0.175 \mathrm{~g}$ of EDMA (17.5 wt \%), $0.5 \mathrm{~g}$ of distilled THF (50 wt \%), and $5 \mathrm{mg}$ of DMPAP (1 wt \% with respect to the monomers). The mixture was sonicated for $45 \mathrm{~min}$, to help dissolve the crystallized Boc-AEMA, and then stirred for $30 \mathrm{~min}$ to achieve complete dissolution. The mixture was used to form a thin network on a flat 20\% X-PMMA substrate by photoinitiated polymerization under UV irradiation. The substrate with the grafted film was then washed with ethanol and dried under nitrogen for $5 \mathrm{~min}$, immersed in a beaker containing $25 \mathrm{~mL}$ of phosphoric acid with stirring for $3 \mathrm{~h}$ for deprotection, washed with ethanol, and dried for 10 $\min$.

\subsection{Monolith formation and characterization}

A mixture consisting of Boc-AEMA (15.6 wt \%), BuMA (1.3 wt \%), EDMA (9.1 wt \%), 1-dodecanol (52.4 wt \%), cyclohexanol (21.6 wt \%), and DMPAP (1 wt \% with respect to the monomers) was introduced into the microchannel, and polymerization was triggered by irradiation for $15 \mathrm{~min}$. The microchannel was then flipped 180 degrees and left under the UV source for $15 \mathrm{~min}$ at $365 \mathrm{~nm} \mathrm{UV}$ wavelength and $200 \mathrm{~mJ} / \mathrm{cm}^{2}$ energy in a cabinet containing a UV lamp (ENF-260C, Spectronics Corp. Westbury, NY, USA) as shown in Scheme 3. 
The microchannel was then flipped 180 degrees and left under the UV source for 15 min longer. Two $30 \mathrm{G}$ syringe needles were trimmed and placed over the inlet and outlet holes. They were set with epoxy glue mixed with fine fumed silica powder, to achieve a hard and stable adhesive layer. Using a Pico plus syringe pump (Harvard apparatus, Holliston, MA USA) the microchannel was flushed with ethanol to remove the unreacted monomers and the porogens.
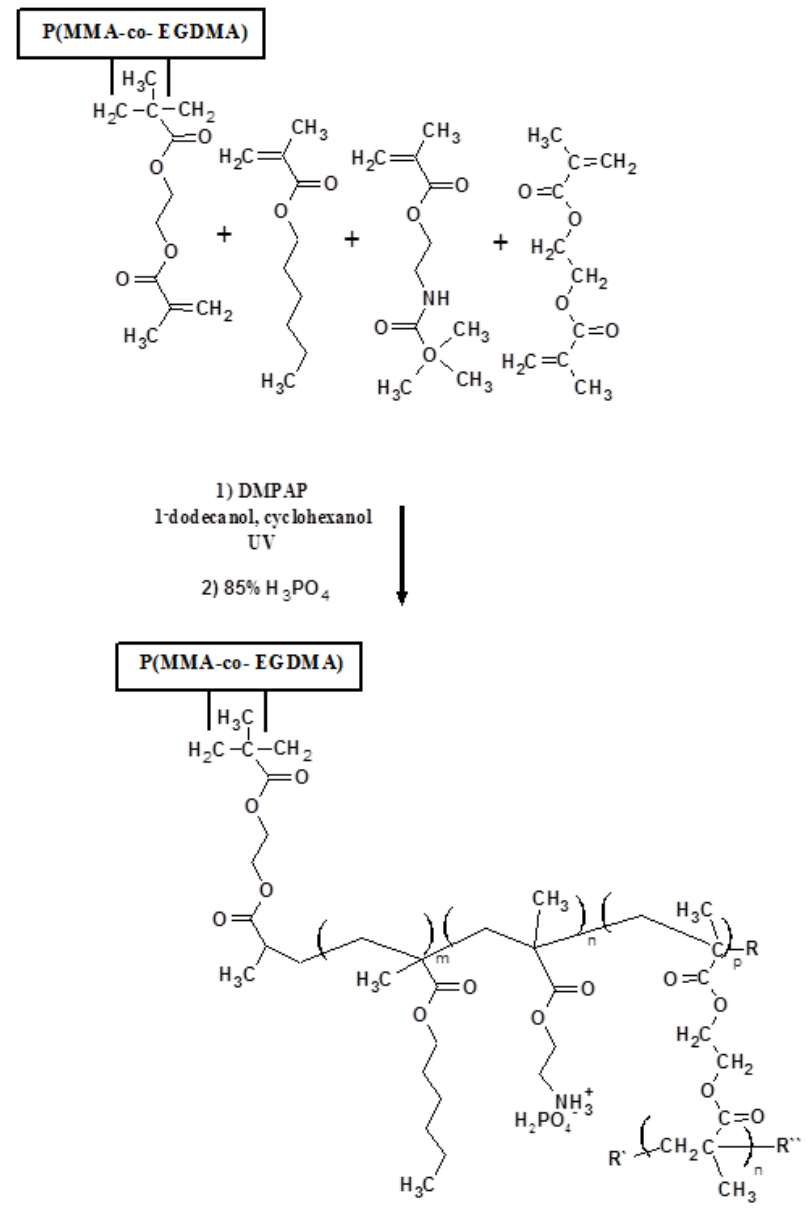

Scheme 3. Reactions for the formation of the monolith column on $20 \%$ X-PMMA surface, and removal of the Boc protecting group with phosphoric acid.

Linear PMMA was first considered as a substrate for the microfluidic channel, but it cracked and gradually dissolved when exposed to the monomers. To overcome this obstacle cross-linked PMMA was used. This approach also ensures that unreacted double bonds on the cross-linked surface improve adhesion between the PPM column and the substrate. Attenuated total reflectance-Fourier transform infrared spectroscopy (ATR-FTIR) was used to confirm the presence of dangling $\mathrm{C}=\mathrm{C}$ double bonds on the surface of the cross-linked
PMMA. Monolith pressure tests were conducted to evaluate the bonding strength between the monolith column and the substrate. To that end the microfluidic chip containing the PPM column was connected to a compressed $\mathrm{N}_{2}$ cylinder that was used to pump ethanol through the monolithic column. The pressure was increased in 10 PSI increments every 5 min to determine the pressure at which the monolithic column started to debond from the substrate.

Images of the monoliths were obtained with a Hitachi SU-70 scanning electron microscope (SEM) (Hitachi HighTechnologies Corporation, Tokyo, Japan) at an acceleration voltage of $10 \mathrm{kV}$. The SEM samples were prepared by immersing the microchannel hosting the PPM column into liquid nitrogen, and then cutting perpendicularly to the monolith-filled X-PMMA channel. Gold was sputtered onto the samples prior to SEM imaging.

\subsection{Activation of the antibacterial column via deprotec- tion}

Deprotection of the Boc-AEMA units was achieved by flowing $250 \mu \mathrm{L}$ of phosphoric acid through the PPM column before flushing with ethanol and deionized (DI) water to remove acid residues. After purging, the PPM column was opened by cutting vertically through the monolith-filled X-PMMA channel with an electrical saw. The column material was then removed by scraping and ground into a fine powder, mixed with $\mathrm{KBr}$ in a 1:50 weight ratio, and dried in a vacuum oven at $60^{\circ} \mathrm{C}$ overnight. $\mathrm{KBr}$ pellets were prepared for analysis by pressing the powder in a dye at $10 \mathrm{kPSI}$ for 3 min on a Carver 3851 Press (Thomas Scientific, Swedesboro, NJ USA). To validate the deprotection process, FT-IR spectra were acquired at room temperature on a Bruker Tensor 27 spectrometer by averaging 64 scans recorded at a rate of $1 \mathrm{scan} / \mathrm{s}$. The wavenumber region scanned was between 400 and $4000 \mathrm{~cm}^{-1}$.

\subsection{Antibacterial activity}

The antibacterial properties of the non-porous network and the microporous monolithic column were investigated by several techniques. The same composition of the functional and cross-linking monomers was used in both the nonporous network and the porous column. E. coli and B. subtilis bacterial cells were selected to evaluate the cell lysis ability of the antibacterial PPM column on gram-negative and gram-positive bacterial cells, respectively

2.7.1 Cell samples. E. coli and B. subtilis cells were grown in lysogeny broth (LB) in an incubator at $37^{\circ} \mathrm{C}$, shaking at 180 RPM and left overnight. The bacterial cells were then washed twice with ID water and then re-suspended in phosphate buffered saline (PBS) before the experiments. The stock cell concentration was adjusted with an Ultrospec 2100 pro 
UV/Visible spectrophotometer (Biochrom Ltd., Cambridge, UK), to prove an optical density (OD) value of 0.6 at $600 \mathrm{~nm}$ $\left(\mathrm{OD}_{600}=0.6\right)$ for the bacteria sample. The cell solutions were then diluted as needed in different experiments.

2.7.2 Non-porous polymeric network. The antimicrobial properties of the deprotected network were evaluated by the LIVE/DEAD Cell Viability Assay (L7012, Molecular Probe, USA), used to directly monitor cell viability. The assay uses two fluorescent nucleic acid stains, SYTO 9 (green) and propidium iodide (PI; red). The SYTO 9 stain penetrates both healthy bacterial cells (with intact membranes) and non-healthy cells; it therefore labels both live and dead bacteria. In contrast propidium iodide penetrates only bacteria with damaged membranes, thus reducing the SYTO 9 fluorescence intensity. Consequently, live bacteria with intact membranes fluoresce green while dead bacteria (with disintegrated membranes) fluoresce red, while the background remains virtually non-fluorescent. Images were captured on a fluorescence microscope (Nikon Eclipse E600FN upright) with a digital camera (Nikon Photometrics Coolsnap EZ 12Bit Monochrome Cooled CCD and NIS-ELEMENTS IMAGING Software) through a dual-band filter, so that both cells with and without intact cell membranes could be visualized simultaneously. SYTO 9 and PI $(0.15 \mu \mathrm{L}$ of each) were mixed on a vortex mixer, $100 \mu \mathrm{L}$ of bacteria suspension were added and mixed, and the stock mixture was incubated for $15 \mathrm{~min}$ utes at $37{ }^{\circ} \mathrm{C}$. A $10 \mu \mathrm{L}$ of bacterial suspension/stains mixture was dropped over the non-porous network and covered with a thin microscope slide. The sandwiched layers were left in contact under the microscope, and fluorescent microscopic images were recorded after zero and $300 \mathrm{~s}$ of contact time.

2.7.3 Porous polymeric monolith column. Cell lysis was confirmed via the Ethidium Bromide (EtBr) Intercalation Assay as an indicator of DNA presence in the cell lysate. When EtBr is exposed to ultraviolet light, it fluoresces with an orange color which intensifies after intercalation in DNA. This assay thus relates the intensity of EtBr fluorescence to the DNA concentration in the cell lysate. The florescence intensity of EtBr after intercalation in the DNA released from the microchannel hosting the porous and antibacterial monolithic column was quantified with a Quanta-Master 4 spectrofluorometer (Photon Technology International, London, ON). A $0.1 \mathrm{~mL}$ aliquot of bacterial cells was suspended in PBS buffer (E. coli and B. subtilis at $\mathrm{OD}_{600}=0.25$ ) and pumped through the PPM column before and after deprotection at a flow rate of $1 \mu \mathrm{L} / \mathrm{min}$, and the cell lysate was collected at the outlet. In this assay $0.02 \mathrm{~mL}$ aliquots of E. coli and B. subtilis lysates were each added to a spectrophotometer cuvette containing 0.380 $\mathrm{mL}$ of DI water and $0.03 \mathrm{~mL}$ of EtBr from a stock solution with a concentration of $0.4 \mathrm{mg} / \mathrm{L}$.

Furthermore, to validate the DNA released as a result of cell lysis and to ensure that the PPM column did not leach any polymerase chain reaction (PCR) inhibitors, the cell lysate was used in PCR. The presence of DNA amplified by PCR was confirmed by the increase in fluorescence intensity of ethidium bromide, and finally by gel electrophoresis. A Bio-Rad gel electrophoresis apparatus served to analyze the PCR products on $1.2 \%$ agarose gel using a DC voltage of $85 \mathrm{~V}$ and a running time of $30 \mathrm{~min}$. The gel was subsequently removed from the chamber and was imaged with a Bio-Rad Doc XR imagining system.

2.7.4 PCR reagents and experimental setup. The PCR reaction was performed in a T100 Thermal Cycler (Bio-RD, Montreal, QC Canada) in a $25 \mu \mathrm{L}$ volume consisting of 300 $\mathrm{nM}$ of forward primer, $300 \mathrm{nM}$ of reverse primer, $200 \mu \mathrm{M}$ of dNTPs, $3.5 \mathrm{mM}$ of magnesium chloride, $0.625 \mathrm{U}$ of iTaq polymerase, $2.5 \mu \mathrm{L}$ of $10 \mathrm{X}$ PCR buffer, and $5 \mu \mathrm{L}$ of the crude lysate collected at the outlet of the microfluidic channel. ${ }^{12}$ The structure of the E. coli ${ }^{13}$ and B. subtilis ${ }^{14}$ primers used in the PCR reaction is provided in Table 2. The PCR tubes were first preheated and incubated at $95^{\circ} \mathrm{C}$ for 3 minutes, and then the PCR thermal cycler was programed to run for 30 cycles with $95^{\circ} \mathrm{C}$ for 30 seconds, $63^{\circ} \mathrm{C}$ for 1 minute, and $73^{\circ} \mathrm{C}$ for 1 minute.

Table 2 Primers used in PCR experiments

\begin{tabular}{ll}
\hline Primer direction & Primer sequence \\
\hline E. coli forward primer & 5-AAAACGGCAAGAAAAAGCAG-3́3 \\
E. coli reverse primer & 5-ACGCGTGGTTACAGTCTTGCG-3 \\
B. subtilis forward primer & 5-AAGAGTTTGATCATGGCTCAG-3̀ \\
B. subtilis reverse primer & 5-AGGAGGTGATCCAACCGCA-⿳亠丷厂 \\
\hline
\end{tabular}

\section{Results and discussion}

\subsection{Monolith formation and bonding to the substrate}

To form the monoliths, two functional monomers: BocAEMA and BuMA, were copolymerized with a third crosslinking monomer in the presence of a porogenic solvent mixture. The functional monomers play a key role in the final product, as they introduce the antibacterial component in the structure. The cross-linking monomer is also necessary to form a network, by contributing to the mechanical stability of the monolith. To form a porous network and also control the pore size, porogenic solvents were used. Since Boc-AEMA was synthesized in the crystallized form, the monomer mixture was sonicated and stirred to form a homogeneous solution prior to polymerization. In order to achieve fast polymerization and complete monomer conversion within approximately $10 \mathrm{~min}$, DMPAP was selected as photoinitiator due to its relatively short half-life $\left(\mathrm{t}^{1 / 2}\right)$ or high decomposition 
rate $\left(\mathrm{k}_{d}\right) \cdot{ }^{15}$ Fig. 1 (left) provides an SEM image for the PPM column packing, showing some pores circled and (right) an overall view of the microchip consisting of the microfluidic channel laser-micromachined onto a 20\% X-PMMA substrate hosting the monolithic column. To facilitate the tube connections between the microfluidic channel and the bacterial suspension reservoir, syringe needles were set over the inlet and outlet of the microfluidic channel and bonded to the surface with a mixture of epoxy glue and fumed silica. The unre-

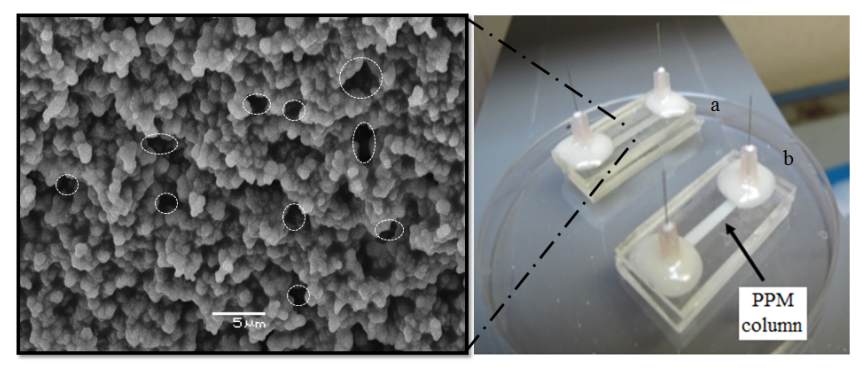

Fig. 1 Microfluidic chip fabricated by laser micromachining on an X-PMMA substrate filled with a monolith column (right) and SEM image of the PPM column packing (left).

acted double bonds on the surface of the cross-linked PMMA substrate allowed covalent bonding with the monolith material and contributed to the mechanical stability of the PPM column within the microfluidic channel, by decreasing the possibility of debonding leading to the creation of voids along the monolithic column. Surface characterization of the crosslinked PMMA sheets was performed by ATR-FTIR to confirm the presence of unreacted carbon-carbon double bonds upon copolymerization of MMA and EGDMA; the spectra obtained are shown in Fig. 2. The absorption band at $1637 \mathrm{~cm}^{-1}$ is characteristic for the $\mathrm{C}=\mathrm{CH}_{2}$ stretching vibration mode vicinal to an ester group. ${ }^{16}$ Also visible is the $\mathrm{C}=\mathrm{CH}_{2}$ out-of-plane bending vibration mode 16 at $949 \mathrm{~cm}^{-1}$. After normalization of the spectra to the $\mathrm{C}=\mathrm{O}$ stretching band $1722 \mathrm{~cm}^{-1}$, the $\mathrm{C}=\mathrm{C}$ absorbance was found to increase slowly with the cross-linker content, as illustrated in the inset of Fig. 2. In contrast to native PMMA, the samples prepared with up to 15 mol\% EGDMA presented evidence for unreacted double bonds at the surface, becoming even more obvious for the sample containing 20 mol $\%$ of EGDMA.

To confirm and quantify bonding between the PPM column and the cross-linked PMMA substrate, a pressure test was used. Table 3 summarizes the pressure withstood by the column before voids appeared along the channel (i.e. between the monolithic column and the substrate), referred to as void pressure, at $3 \%, 5 \%, 10 \%, 15 \%$ and $20 \%$ cross-linker content. The results in Table 3 are consistent with improved bonding or anchoring of the column material as the cross-linking level of the substrate increased.

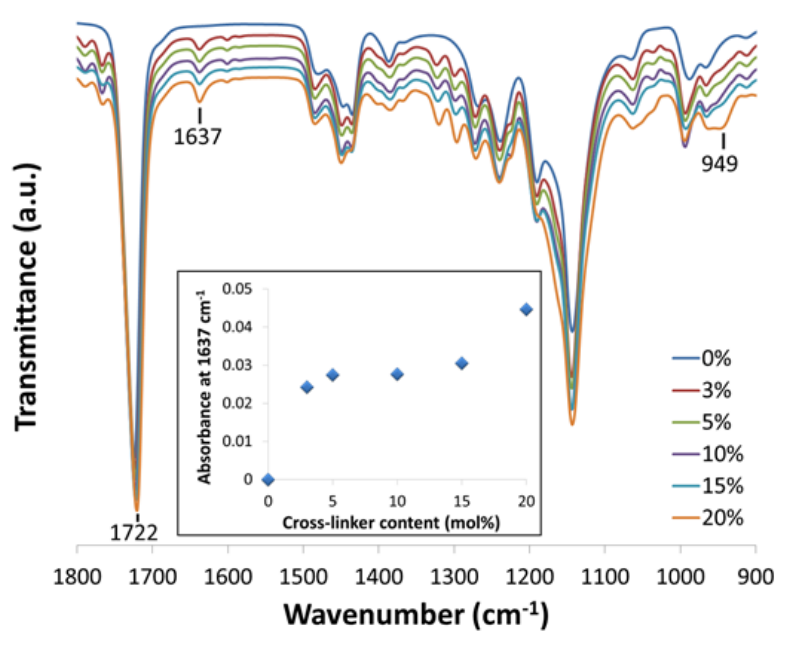

Fig. 2 ATR-FTIR spectra for native and cross-linked PMMA samples with cross-linker contents ranging from 3 to $20 \mathrm{~mol} \%$. The vibrational modes at 1637 and $949 \mathrm{~cm}^{-1}$ correspond to $\mathrm{C}=\mathrm{CH} 2$ stretching and out-of-plan bending, respectively. Inset: Absorbance at $1637 \mathrm{~cm}^{-1}$ for the different X-PMMA samples.

Table 3 Debonding pressure of the column from the substrate for different cross-linking levels.

\begin{tabular}{ll}
\hline Cross-linker (mole \%) & Void pressure (psi) \\
\hline 3 & 50 \\
5 & 60 \\
10 & 80 \\
15 & 100 \\
20 & 150 \\
\hline
\end{tabular}

\section{$3.2 t$-Boc group deprotection}

Several activation methods were investigated to optimize the antibacterial activity of the PPM column. A mixture of hydrochloric acid and dioxane (1:2 ratio) was first used, but the mixture dissolved the plastic tip of the syringe needle and degraded the connections tubes, which made it unsatisfactory for deprotecting the amine group in the Boc-AEMA monomer. Trifluoroacetic acid (TFA) was also investigated but it was likewise aggressive on the syringe, the needle, and the tubes and it slowly eroded the PPM column; therefore this deprotection method was likewise discarded early on. Phosphoric acid was finally preferred to deprotect the Boc-AEMA units. Deprotection was successfully achieved by flowing phosphoric acid through the column, as evidenced by FT-IR analysis (Fig. 3).

The absorption bands characteristic for the carbamate functional group at $1518 \mathrm{~cm}^{-1}$, corresponding to the $-\mathrm{N}-\mathrm{H}$ bending vibrational mode, decreased significantly in the spectrum after the deprotection reaction and a small broad peak visi- 
ble at $1541 \mathrm{~cm}^{-1}$ appeared, corresponding to the $-\mathrm{NH}_{2}$ bending mode. A weaker absorbance associated with the N-CO$\mathrm{O}$ symmetrical stretching mode at $872 \mathrm{~cm}^{-1}$ was also observed ${ }^{17,18}$. Additionally, the peak for the $\mathrm{CH}_{3}$ bending mode of the tert-butyl group at $1367 \mathrm{~cm}^{-1}$ decreased in intensity. ${ }^{18}$

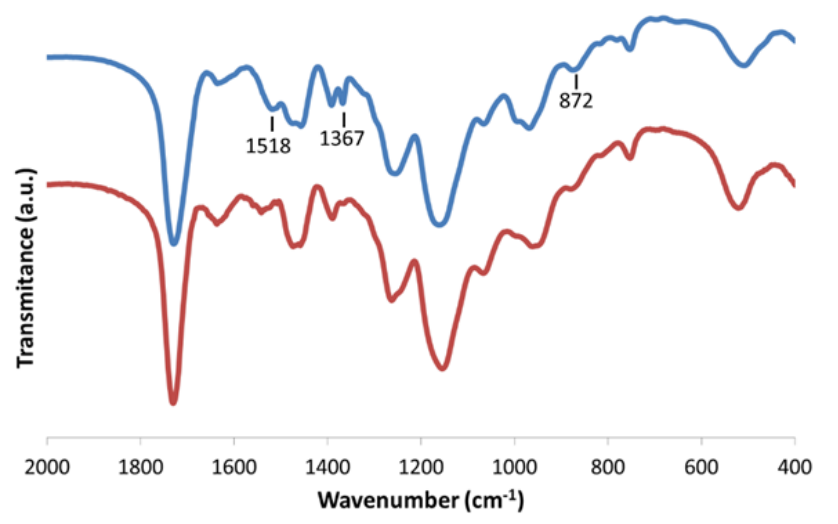

Fig. 3 FT-IR spectra for the PPM before (top) and after (bottom) deprotection of the t-Boc group. Vibrational band assignments: $\mathrm{N}-\mathrm{CO}-\mathrm{O}$ symmetrical stretching at $1518 \mathrm{~cm}^{-1}, \mathrm{CH}_{3}$ bending at $1367 \mathrm{~cm}^{-1}$, N-CO-O symmetrical stretching at $872 \mathrm{~cm}^{-1}$.

\subsection{Antibacterial activity of the non-porous network}

Bacteria viability was monitored by the double staining technique described in Section 2.7.2. The green fluorescence is gradually replaced with red fluorescence in these experiments, as shown in Fig. 4A and B, providing clear evidence for membrane disintegration and cell lysis. In contrast to the deprotected network, the protected network did not display any lysis ability, as shown in Fig. 4C and D. The red and green fluorescence intensities were recorded for up to $300 \mathrm{~s}$ as shown in Fig. 5. For the deprotected network, the green and red intensities decreased and increased with time, respectively, as illustrated in Fig. 5, reflecting the fact that the cell membranes slowly became permeable, thus allowing the penetration of propidium iodide and reducing the fluorescence intensity for SYTO 9. This provides clear evidence that the non-porous network became antibacterial once the t-Boc protecting group was removed. For the protected column, in contrast, the green and red fluorescence intensities remained almost constant as shown in Fig. 5, reflecting the fact that protected network does not show any antibacterial activity.

\subsection{Antibacterial activity of the PPM column}

To validate semi-quantitatively the DNA released after lysing the bacterial cells by flowing them through the antibacterial porous medium of the PPM column, ethidium bromide was
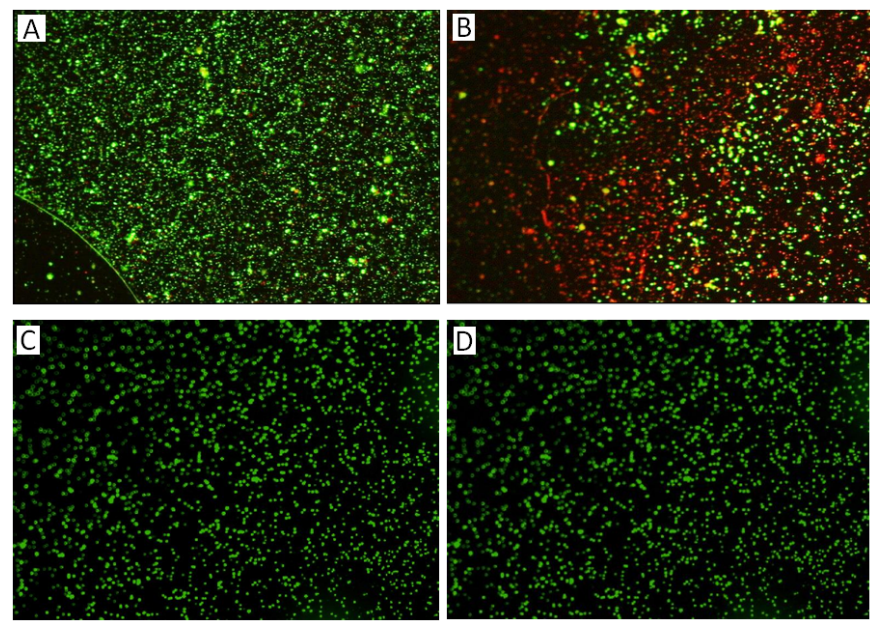

Fig. 4 Fluorescence intensities for E. coli suspended in PBS buffer and stained with Live/Dead dye in contact with (A) the deprotected antibacterial non-porous network at time zero and (B) after $300 \mathrm{~s}$, and in contact with $(\mathrm{C})$ the protected non-porous network at time zero and (D) after $300 \mathrm{~s}$.

used as an indicator of DNA presence. Fig. 6 illustrates the effect of the released DNA on the EtBr fluorescence intensity after flowing E. coli and B. subtilis bacterial cells through the PPM column before and after deprotection, and also after amplifying the collected DNA with PCR. The figure clearly shows that the fluorescence intensity of $\mathrm{EtBr}$ increased when adding the lysate collected after flowing the bacterial cells through the narrow porous channel of the monolithic column, even for the packing material in its protected form, confirming that the bacterial cells were partly lysed through shearing and their DNA was released. However it can also be seen that the fluorescence intensity of EtBr further increased for the cell lysate collected after flowing the bacterial cells through the monolithic column in its deprotected form, thus confirming that an incremental amount of cell lysis was achieved by physical contact of the cells with the antibacterial surface, resulting in the release of more DNA. The fluorescence intensity of EtBr further increased for the PCR output, which confirms that PCR was not strongly inhibited by the cell lysate eluted from the column and that the DNA genes of E. coli and B. subtilis could be successfully amplified. The increases observed (ca. 2- to 3-fold after 30 amplification cycles) remain rather modest, and it is not clear whether this is due to the release of minute amounts of PCR inhibitors by the PPM column or to the presence of interfering proteins in the crude cell lysate used in the amplification process. ${ }^{19}$ The determination of the exact origin of these modest increases will be the topic of a future investigation.

Moreover, the DNA released from both E. coli and B. subtilis amplified by PCR was qualitatively validated by gel elec-

This journal is @ The Royal Society of Chemistry [year]

Journal Name, 2010, [vol], 1-9 |7 


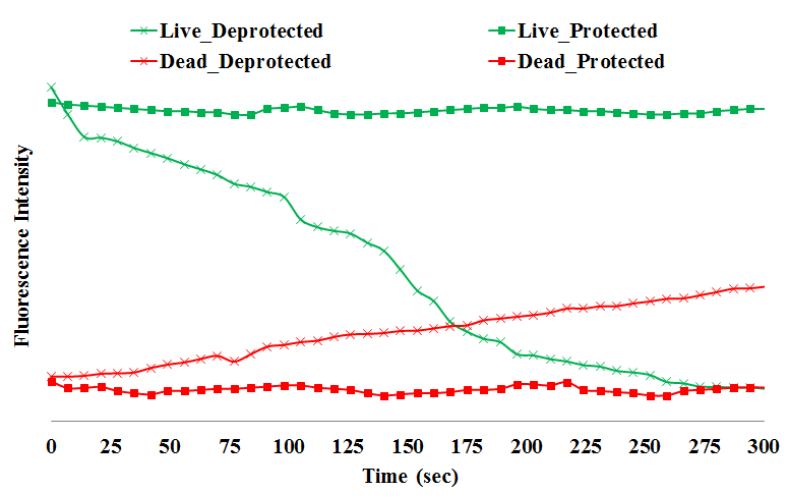

Fig. 5 Green (Live) and red (Dead) stain intensities with respect to contact time before and after removing the Boc protecting group.

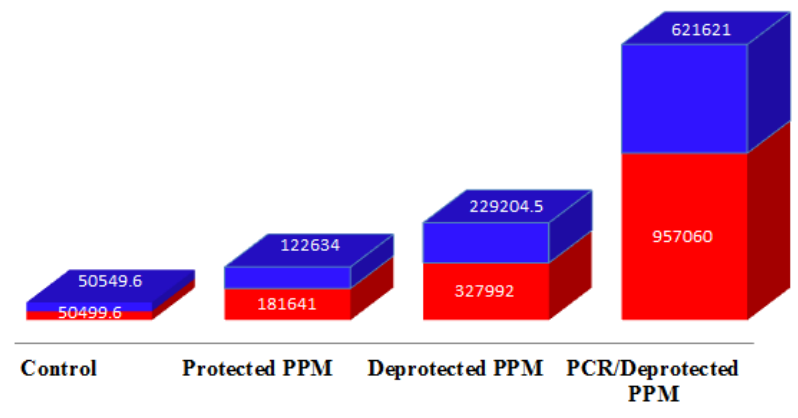

Fig. 6 Fluorescence intensity of EtBr after adding E. coli (blue) and B. subtilis (red) cells suspended in PBS buffer (control), the lysate of E. coli and B. subtilis suspensions flown through the PPM column before (Protected PPM) and after deprotection (Deprotected PPM), and PCR-amplified product from the Deprotected PPM cell lysate (PCR/Deprotected PPM).

trophoresis. Fig. 7 shows the gel electrophoresis analysis results for the PCR products of B. subtilis bacterial cells that were not flown through the porous column (column 2), B. subtilis lysate collected at the outlet of the deprotected PPM column (column 3), E. coli lysate collected at the outlet of the deprotected monolithic column before (column 4) and after (column 5) filtration using a $0.2 \mu \mathrm{m}$ filter, and an E. coli bacterial suspension that was not flown through the porous column (column 6). Fig. 7 shows no detectable amount of DNA at the PCR output for the E. coli and B. subtilis samples that were not flown into the monolithic column, which confirms that the bacterial cells had intact cell membranes before passing through the antibacterial monolithic columns. On the other hand, DNA is clearly detected at the PCR output for the E. coli and B. subtilis samples that were flown through the deprotected PPM columns, which confirms that the membranes of bacterial cells were damaged and disintegrated by flowing through the PPM column. It can also be observed that filtration of the cells before running the PCR did not affect the amplification procedure. This shows that the porous column lysed the bacterial cells and filtered the cell debris and any intact cells left in the samples.

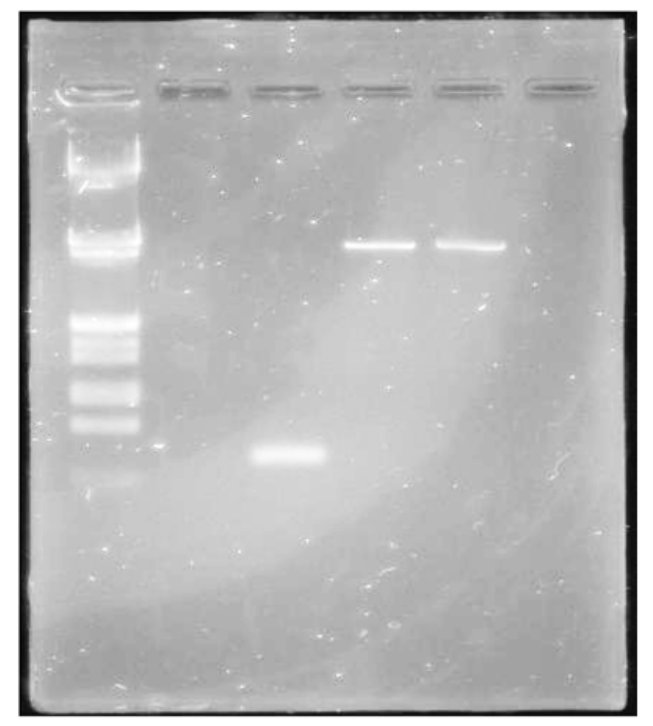

Fig. $7 \mathrm{Gel}$ electrophoresis analysis of PCR output for unlysed (column 2) and lysed (through PPM) (column 3) B. subtilis cells, filtered (column 4) and nonfiltered (column 5) lysed E. coli and nonlysed E. coli (column 6). Column 1 is for a 100 bp DNA ladder.

\section{Conclusions}

It has been demonstrated that the activated antibacterial porous columns prepared have the ability to effectively lyse E. coli and B. subtilis bacterial cells, and to yield DNA that is ready to use in PCR experiments without further cleaning or filtration. The combination of shear degradation and antibacterial properties was clearly beneficial to achieve cell lysis, which was evidenced by the release of DNA. Cell lysis was confirmed by several techniques, namely the LIVE/DEAD Cell Viability Assay that was used to directly monitor cell viability on the nonporous network, the Ethidium Bromide Intercalation Assay utilizing spectrofluorometry, relating the DNA concentration to the increase in fluorescence intensity of $\mathrm{EtBr}$, as well as by gel electrophoresis to validate the PCR amplification test. The usefulness of X-PMMA as a substrate on which the microfluidic channel could be laser-micromachined without further surface functionalization was also demonstrated in this work. The PPM formed on 20\% X-PMMA could withstand $150 \mathrm{psi}$ before voids started to appear across the monolithic column. 


\section{Acknowledgements}

We would like to express our gratitude to Grand Challenges Canada and Bigtec Labs for their financial support. We also like to thank Prof. Michael Palmer, Mr. Mohamed Salah, and Mr. Eric K. Brefo-Mensah, from the Department of Chemistry at the University of Waterloo, for providing access to their lab facilities and their guidance on some of the biological concepts.

\section{References}

1 M. Mahalanabis, H. Al-Muayad, M. D. Kulinski, D. Altman and C. M. Klapperich, Lab Cip, 2009, 9, 2811-2817.

2 A. Bhattacharyya, D. Kulinski and C. Klapperich, J. Vis. Exp., 2008, 12, 664.

3 K. Lienkamp, A. E. Madkour, A. Musante, C. F. Nelson, K. Nusslein and G. N. Tew, J. Am. Chem. Soc., 2008, 130, 9836-9843.

4 J. C. Tiller, C.-J. Liao, K. Lewis and A. M. Klibanov, J. Am. Chem. Soc., 2001, 98, 5981-5985.

5 R. Advincula, in Surface-Initiated Polymerization I, ed. R. Jordan, Springer Berlin Heidelberg, 2006, pp. 107-136.

6 S. Edmondson, V. L. Osborne and W. T. S. Huck, Chem. Soc. Rev., 2003, 33, 14-22.

7 P. T. Hammond, AIChE J., 2011, 57, 1547-5905.

8 J. Lyklema and L. Deschenes, Adv. Colloid Interface Sci., 2011, 168, 1547-5905.

9 A. E. Madkour, J. M. Dabkowski, K. Nusslein and G. N. Tew, Langmuir, 2008, 25, 1060-1067.

10 I. A. Fedotenko, P.-L. Zaffalon, F. Favarger and A. Zumbuehl, Tetrahedron Lett., 2010, 51, 5382-5384.

11 K. Kuroda and W. F. DeGrado, J. Am. Chem. Soc., 2005, 127, 4128-4129.

12 W. Wan and J. Yeow, Biomed. Microdevices, 2011, 14, 337-346.

13 A. K. BEJ, J. L. DiCESARE, L. HAFF and R. M. ATLAS, Appl. Environ. Microbiol., 1991, 57, 1013-1017.

$14 \mathrm{H}$. Abushady, A. Bashandy, N. Aziz and H. Ibrahim, Intern. J. of Agric. and Biol., 2005, 7, 337-344.

15 A. Bhattacharyya and C. M. Klapperich, Anal. Chem., 2006, 78, 788-792.

16 E. Pretsch, T. Clerc, J. Seibl and W. Simon, Tables of spectral data for structure determination of organic compounds, Springer: Berlin, 1989.

17 E. Pretsch, P. Buhlmann and M. Badertscher, Structure Determination of Organic Compounds, Springer: Berlin, 4th edn., 2009, p. 269 and 324.

18 S. G. Roy, R. Acharya, U. Chatterji and P. De, Poly. Chem., 2013, 4, $1141-1152$.

19 P. Morata, M. I. Queipo-Ortuno and J. D. D. Colmenero, J.Clin.Microbiol., 1998, 36, 2443-2446.

This journal is @ The Royal Society of Chemistry [year]

Journal Name, 2010, [vol], 1-9|9 